\title{
Excitonic Quasimolecules in Nano Systems of Semiconductor and Dielectric Quantum Dots
}

Sergey I Pokutnyi*

Chuiko Institute of Surface Chemistry, National Academy of Sciences of Ukraine, 17 General Naumov Str. Kyiv UA 03164, Ukraine

\begin{abstract}
A review devoted to the theory of excitonic quasimolecules (Biexcitons) (formed of spatially separated electrons and holes) in a nanosystems that consists of semiconductor and dielectric quantum dots synthesized in a dielectric matrix. It is shown that exciton quasimolecules formation is of the threshold character and possible in nanosystems, in with the spacing between the quantum dots surfaces is larger than a certain critical spacing. It was found that the binding energy of singlet ground state of exciton quasimolecules, consisting of two semiconductor quantum dots is a significant large values, larger than the binding energy of the biexciton in a semiconductor single crystal almost two orders of magnitude.
\end{abstract}

Keywords: Spatially separated electron and holes; Quantum dots; Binding energy; Coulomb and exchange interaction

\section{Introduction}

The idea of superatom was fruitful for the development of nanophysics [1-3] Superatom (quasiatomic nanoheterostructures) consists of a spherical quantum $\operatorname{dot}(\mathrm{QD})$ with radius $a$, the volume of that contains the semiconductor (or dielectric) material. QD is surrounded by dielectric (semiconductor) matrix [2,3]. A hole is localized in the volume of QD, and the electron is localized over a spherical interface (QD-matrix). In this nanosystem the lowest electronic level is in matrix, and the lowest hole level is within volume of QD. A large shift of the valence band $(700 \mathrm{meV})$ generates the localization of holes in the volume of QD. A significant shift of the conduction band (about 400 $\mathrm{meV}$ ) is a potential barrier for electrons [4] (Figure 1). The electrons moving in the matrix and do not penetrate in the volume of QD [2-4]. The energy of the Coulomb interaction of electron with hole and the energy of the polarization interaction of electron with interface (QDmatrix) form a potential well, in which the electron is localized over the surface of quantum dot. The certain orbitals, localized surrounding quantum dot correspond to electrons in superatom [2,3]. During investigation of the optical characteristics of nanosystems with CdS,
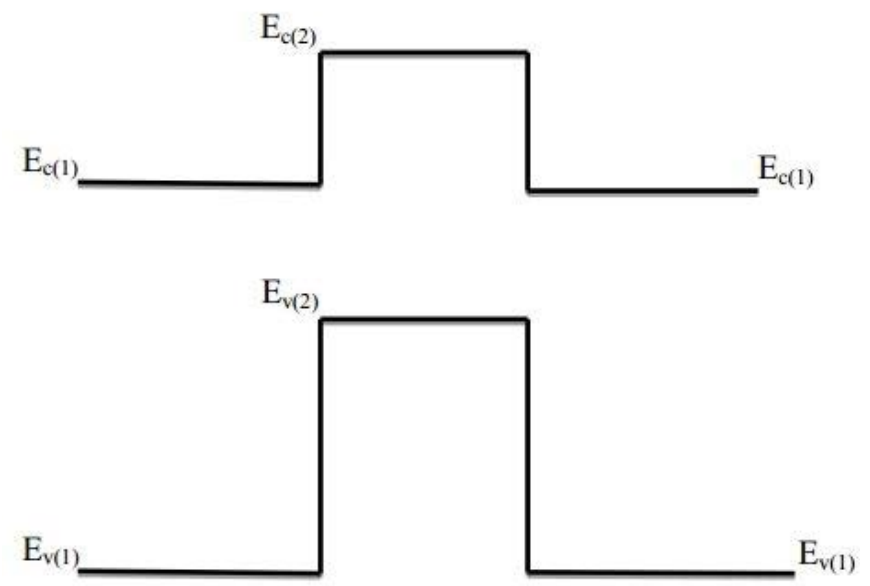

Figure 1: Band diagram of the QD - matrix nano hetero structure. In the nano hetero structure, the QD is a potential well for a hole and a barrier for an electron. The energies $E_{\text {(1) }}, E_{v(1)}, E_{\text {a }}$ and $E_{\text {(2) }}$, correspond to the positions of the bottom of the conduction band and the top of the valence band of the matrix and QD, respectively.
$\mathrm{ZnSe}, \mathrm{Al}_{2} \mathrm{O}_{3}$ and $\mathrm{Ge}$ quantum dots in experimental papers [4-8] it was found that the electron can be localized above the surface of the QD while the hole here moves in the volume of the QD. In Ref. [4-8] the appearance of super atoms located in dielectric matrices as cores containing $\mathrm{CdS}, \mathrm{ZnSe}, \mathrm{Al}_{2} \mathrm{O}_{3}$ and $\mathrm{Ge}$ quantum dots was apparently established experimentally for the first time. A substantial increase in the bond energy of the ground state of an electron in a super atom in comparison with the bond energy of an exciton in CdS, $\mathrm{ZnSe}$ and $\mathrm{Al}_{2} \mathrm{O}_{3}$ and single crystals was detected in Ref. [2-10].

In Ref. [5-8] the optical characteristics of samples of borosilicate glasses doped with $\mathrm{CdS}, \mathrm{ZnSe}$ and $\mathrm{Al}_{2} \mathrm{O}_{3}$ at concentrations between $x \approx 0.003 \%$ to $1 \%$ were investigated. The average radii $\bar{a}$ of CdS and ZnSe QDs were in the range of $\bar{a} \approx 2.0-20 \mathrm{~nm}$. When there were large concentrations of CdS quantum dots in the samples (from $x \approx 0.6 \%$ to $x$ $\approx 1 \%)$ a maximum, interpreted by the appearance of bonded QD states, was detected in the low-temperature absorption spectra. In order to explain the optical characteristics of such nanosystems we proposed a model of a quasimolecules representing two ZnSe and CdS QDs that form an exciton quasimolecule as a result of the interaction of electrons and holes [3,11-14].

It was noted Ref. [4-6] that, at such a QD content in the samples, one must take into account the interaction between charge carriers localized above the QD surfaces. Therefore, in Ref. [11-14], we develop the theory of a exciton quasimolecule (or biexciton) (formed from spatially separated electrons and holes) in a nanosystems that consists of ZnSe and CdS QDs synthesized in a borosilicate glassy matrix. Using the variational method, we obtain the total energy and the binding energy of the exciton quasimolecule (or biexciton) singlet ground state in such system as functions of the spacing between the QD surfaces and of the QD radius. We show that the biexciton formation is of the threshold character and possible in a nanosystems, in which the

*Corresponding author: Sergey I Pokutnyi, Chuiko Institute of Surface Chemistry, National Academy of Sciences of Ukraine, 17 General Naumov Str. Kyiv UA 03164, Ukraine, Tel: 380442396666; E-mail: Pokutnyi_Sergey@inbox.ru (or) pokytniy@mail.ua

Received September 02, 2016; Accepted September 14, 2016; Published September 20, 2016

Citation: Pokutnyi SI (2016) Excitonic Quasimolecules in Nano Systems of Semiconductor and Dielectric Quantum Dots. Mod Chem appl 4: 188. doi: 10.4172/2329-6798.1000188

Copyright: () 2016 Pokutnyi SI. This is an open-access article distributed under the terms of the Creative Commons Attribution License, which permits unrestricted use, distribution, and reproduction in any medium, provided the original author and source are credited. 
spacing between the QD surfaces exceeds a certain critical spacing. It is established that the spectral shift of the low-temperature luminescence peak [6] in such a nanosystem is due to quantum confinement of the energy of the biexciton ground state.

The convergence of two (or more) QDs up to a certain critical value $D c$ between surfaces of QD lead to overlapping of electron orbitals of superatoms and the emergence of exchange interactions [3,11-14]. In this case the overlap integral of the electron wave functions takes a significant value. As a result, the conditions for the formation of quasimolecules from QDs can be created [3,11-14]. One can also assume that the above conditions of formation of quasimolecules can be provided by external physical fields. This assumption is evidenced by results of Ref. $[15,16]$ in which the occurrence of the effective interaction between QDs at considerable distances under conditions of electromagnetic field was observed experimentally. In Ref. [17] energies of the ground state of "vertical" and "horizontal" located pair of interacting QDs ("molecules" from two QDs) were determined as a function of the steepness of the confining potential and the magnetic field strength. The quantum part of nanocomputer, which was implemented on a pair of QDs ("molecules" from two QDs) with charge states is $n$ qubits [18]. The first smoothly working quantum computer has been on QDs with two electron orbital states as qubits, described by a pseudospin $1 / 2$. As a single cell was taken a couple of asymmetric pair QDs with different sizes and significantly different own energy. The electron, injected into the heterostructure from the channel occupied the lower level. That is, it was located in a QD with larger size. The review deals with the theory of excitonic quasimolecules (biexcitons) (formed of spatially separated electrons and holes) in a nanosystems that consists of semiconductor quantum dots synthesized in a borosilicate glass matrix. It is shown that exciton quasimolecule formation is of the threshold character and possible in nanosystem, in with the spacing between the quantum dots surfaces is larger than a certain critical spacing. It was found that the binding energy of singlet ground state of exciton quasimolecule, consisting of two semiconductor quantum dots is a significant large values, larger than the binding energy of the biexciton in a semiconductor single crystal almost two orders of magnitude.

\section{Binding Energy of Singlet Ground State of Excitonic Quasimolecule}

Consider the model of nanosystems [3,11-14], containing of two superatom. In this model superatoms consist from spherical semiconductor QDs, $A$ and $B$, synthesized in a matrix of borosilicate glass with dielectric constant $\varepsilon_{1}$. Let the QD radii be $a$, the spacing between the spherical QD centers be $L$, and the spacing between the spherical QD surfaces be $D$. Each QD is formed from a semiconductor material with dielectric constant $\varepsilon_{2}$ For simplicity and without loss of generality, we assume that holes $h(A)$ and $h(B)$ with effective masses $m_{h}$ are localized in centers of QD $(A)$ and $\mathrm{QD}(B)$ and electrons $e(1)$ and $e(2)$ with effective masses $m_{e}^{(1)}$ are localized near the spherical surfaces of $\mathrm{QD}(A)$ and $\mathrm{QD}(B)$, respectively $\left(r_{\mathrm{A}(1)}\right.$ is the distance of the electron $e(1)$ from the $\mathrm{QD}(\mathrm{A})$ center; $\left(r_{\mathrm{B}(2)}\right.$ is the distance of the electron $e(2)$ from the $\mathrm{QD}(\mathrm{B})$ center; $\left(r_{\mathrm{A}(2)}\right.$ is the distance of the electron $e(2)$ from the $\mathrm{QD}(\mathrm{A})$ center; $\left(r_{\mathrm{B}(1)}\right.$ is the distance of the electron $e(1)$ from the $\mathrm{QD}(\mathrm{B})$ center; $r_{12}$ is the distance between the electron $e(1)$ and $e(2)$ (Figure 2). The above assumption is reasonable, since the ratio between the effective masses of electron and hole in the nanosystem is much smaller than unity $\left.\left(\left(m_{\mathrm{e}}{ }^{(1)}\right) / m_{\mathrm{h}}\right)<<1\right)$. Let us assume that there is an infinitely high potential barrier on the spherical QD - matrix interface. In the nanosystem the holes do not therefore escape from the volume of the QD while the electrons do not enter the QD.

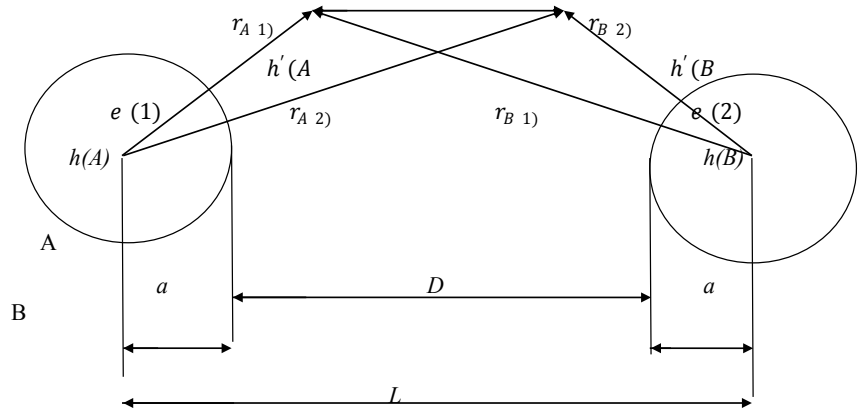

Figure 2: Schematic representation of a nano system consisting of two spherical QDs, QD(A) and QD(B) of radii a. The holes $h(A)$ and $h(B)$ are located in the $\mathrm{QD}(\mathrm{A})$ and $\mathrm{QD}(\mathrm{B})$ centers, and the electrons $e(1)$ and $e(2)$ are localized near the $\mathrm{QD}(\mathrm{A})$ and $\mathrm{QD}(\mathrm{B})$ surfaces $r_{A(1)}$ is the distance of the electron $e(1)$ from the QD(A) center; $r_{B(2)}$ is the distance of the electron $e(2)$ from the QD(B) center; $\boldsymbol{r}_{A(2)}$ is the distance of the electron e(2) from the QD(A) center; $\boldsymbol{r}_{B(1)}$ is the distance of the electron $e(1)$ from the $\mathrm{QD}(\mathrm{B})$ center; $\boldsymbol{r}_{12}$ is the spacing between the electrons $e(1)$ and $e(2) ; L$ is the spacing between the QD centers; $D$ is the spacing between the QD surfaces; $e^{\prime}(1), e^{\prime}(2)$ and $h^{\prime}(A), h^{\prime}(B)$ are the image charges of the electrons and holes.

In such a model of the nanosystem we will study the possible formation of an exciton quasimolecule from spatially confined electrons and holes. The holes are located at the centers of QD(A) and $\mathrm{QD}(\mathrm{B})$, while the electrons are localized close to the spherical surfaces of $\mathrm{QD}(\mathrm{A})$ and $\mathrm{QD}(\mathrm{B})$. In terms of the adiabatic approximation and also in the effective mass approximation we write the Hamiltonian of such a quasimolecule in the following form [3-11].

$$
\hat{H}=\hat{H}_{A(1)}+\hat{H}_{B(2)}+\hat{H}_{i n t}
$$

Here $\hat{H}_{A(1)}$ and $\hat{H}_{B(2)}$ are the Hamiltonians of the superatoms. The hole $h(A)$ is at the center of $\mathrm{QD}(A)$ while the electron $e(1)$ is localized above the surface of $\mathrm{QD}(\mathrm{A})$; the hole $h(\mathrm{~B})$ is located at the center of $\mathrm{QD}(\mathrm{B})$ while the electron $e(2)$ is localized above the surface of $\mathrm{QD}(\mathrm{B})$ respectively. The Hamiltonian of the superatom $\hat{H}_{A(1)}$ will therefore take the following form [3-11]

$$
\widehat{H}_{A(1)}=-\frac{h^{2}}{2 \mu} \Delta_{(1)}+V_{e(1) h(A)}\left(\boldsymbol{r}_{A(1)}, \boldsymbol{r}_{\boldsymbol{h}(A)}\right)+U\left(\boldsymbol{r}_{A(1)}, \boldsymbol{r}_{\boldsymbol{h}(A)}, a\right)+V_{e}(1)\left(r_{A}(1)\right)+V_{h(A)}\left(r_{h(A)}\right)+E_{g,}(2)
$$

In (2), the first term is the kinetic energy operator of the exciton; the energy of Coulomb interaction $\mathrm{V}_{\mathrm{e}(1) \mathrm{h}(\mathrm{A})}$ between the electron $\mathrm{e}(1)$ and the hole $\mathrm{h}(\mathrm{A})$ is described by the formula [2]:

$$
V_{e(1) h(A)}=-\frac{1}{2}\left(\frac{1}{\varepsilon_{1}}+\frac{1}{\varepsilon_{2}}\right) \frac{e^{2}}{\left|\boldsymbol{r}_{e(1)}-\boldsymbol{r}_{\boldsymbol{h}(\boldsymbol{A})}\right|}
$$

the potentials

$$
\begin{aligned}
& V_{h(A)}\left(r_{h(A)}\right)=\left\{\begin{array}{l}
0, r_{h(A)} \leq a \\
\infty, r_{h(A)}>a
\end{array}\right. \\
& V_{e(1)}\left(r_{A(1)}\right)=\infty, \mathrm{r}_{\mathrm{e}(1)} \leq a
\end{aligned}
$$

describe the motion of quasiparticles in the nanosystem in the model of an infinitely deep potential well; and $E_{g}$ is the band gap in the semiconductor with the permittivity $\varepsilon_{2}$. In [2] in the context of the modified effective mass method [19] the theory of an exciton formed from an electron and a hole spatially separated from the electron was developed (the hole was in motion within the QD and the electron was localized on the outer side of the spherical QD-matrix interface). In [2], the energy of the polarization interaction of the electron and hole with the spherical interface with the relative permittivity $\left(\varepsilon=\left(\varepsilon_{2} / \varepsilon_{1}\right)<<1\right)$, $U\left(r_{\mathrm{A}(1)}, r_{h(A)}, a\right)$, is represented as the algebraic sum of the energies of 
interaction of the hole $h(A)$ and the electron $e(1)$ with their own $\left(\mathrm{V}_{\mathrm{h}(A)}\right.$ $\mathrm{h}^{\prime}(A) \mathrm{V}_{\mathrm{e}(1) \mathrm{e}^{\prime}(1)}$ and foreign $\mathrm{V}_{\mathrm{e}(1) h(A)} \mathrm{V}_{\left.\mathrm{h}(1) \mathrm{e}^{\prime}\right)}$ images:

$$
\begin{aligned}
& U\left(r_{\mathrm{A}(1)}, r_{h(A)}, a\right)=+\left(r_{h(A)}, a\right)+\mathrm{V}_{\mathrm{e}(1) \mathrm{e}^{\mathrm{e}}(1)(} r_{\mathrm{A}(1), a)+} \mathrm{V}_{\mathrm{h}(A) \mathrm{e}^{\mathrm{e}(1)}}\left(r_{h(A)}, r_{\mathrm{A}(1),}, a_{,)+} \mathrm{V}\right. \\
& \left(r_{\mathrm{A}(1)}, r_{h(A)}, a\right) \\
& V_{e(1) e^{\prime}(1)}=-\frac{e^{2} \beta}{2 \varepsilon_{2} a} \cdot \frac{a^{2}}{a^{2}-r_{\mathrm{A}(1)}^{2}}+\varepsilon \\
& V_{e(1) e^{\prime}(1)}=-\frac{e^{2} \beta}{2 \varepsilon_{1} a} \cdot \frac{a^{4}}{r_{\mathrm{A}(1)}^{2}\left(r_{\mathrm{A}(1)}^{2}-a^{2}\right)^{\prime}} \\
& V_{h(\mathrm{~A}) e^{\prime}(1)}=-\frac{e^{2} \beta}{2 \varepsilon_{2} a} \cdot \frac{a^{2}}{r_{\mathbf{A}(1)} \mid r_{h(\mathbf{A})}-\left(a / r_{\mathbf{A}(1)}\right)^{2} r_{\mathbf{A}(1)}} \\
& V_{e(1) h^{\prime}(\mathrm{A})}=-\frac{e^{2} \beta}{2 \varepsilon_{1} a} \cdot \frac{a^{2}}{r_{\boldsymbol{h}(\mathbf{A})}\left|r_{\mathbf{A}(1)}-\left(a / r_{h(A)}\right)^{2} r_{h(A)}\right|^{\bullet}}
\end{aligned}
$$

Here $r_{\mathrm{h}(\mathrm{A})}$ is the distance of the hole from the $\mathrm{QD}(\mathrm{A})$ center.

In the quasimolecule Hamiltonian (1), $\hat{\mathrm{H}}_{\mathrm{B}(2)}$ is the Hamiltonian of an exciton formed from an electron and a hole spaced from the electron (the hole $\mathrm{h}(\mathrm{B})$ is located in the QD (B) center and the electron e(2) is localized above the $\mathrm{QD}(\mathrm{B})$ surface). The Hamiltonian $\hat{\mathrm{H}}_{\mathrm{B}(2)}$ has a form similar to that of the Hamiltonian $\hat{\mathrm{H}}_{\mathrm{A}(1)}$ in (2):

$$
\hat{H}_{B(2)}=\frac{\hbar^{2}}{2 \mu} \Delta_{(2)}+V_{e(2) h(B)}\left(\left(r_{B(2)}, r_{h(B)}\right)+U\left(r_{B(2)}, r_{h(B)}, a\right)\right)+V_{e(2)}\left(r_{B(2)}\right)+V_{h(B)}\left(r_{h(B)}\right)+E_{g}
$$

The terms entering into the Hamiltonian (11) are expressed by formulas similar to the corresponding formulas in the Hamiltonian (2). Let us write the expression for the Hamiltonian $\hat{H}_{\text {int }}$ [3-11]

$$
\hat{H}_{i n t=} \mathrm{V}_{A B(D, a)+} \mathrm{V}_{\mathrm{e}(1) h(B)}\left(r_{B(1)}, r_{\mathrm{h}(\mathrm{B})}\right)+\mathrm{V}_{\mathrm{e}(2) h(A))}\left(r_{\mathrm{A}(2)}, r_{h(A)}+\mathrm{V}_{\mathrm{e}(1) \mathrm{e}(2)}\left(r_{12}\right)\right.
$$

Here, $\mathrm{V}_{A B(D, a)}$ is the energy of the interaction of charge carries (the electrons $e(1)$ and $e(2)$ and the holes $h(A)$ and $h(B)$ ) with polarization fields induced by these charge carries at the $\mathrm{QD}(\mathrm{A})$ and $\mathrm{QD}(\mathrm{B})$ surfaces:

$\mathrm{V}_{A B(D, a)=}\left(\mathrm{V}_{\mathrm{h}(A) \mathrm{h}(B)}\right)^{(\mathrm{D}, a)}+\left(\mathrm{V}_{\mathrm{h}(A) \mathrm{h}^{\prime}(B)}\right)^{(D, a)}+\left(\mathrm{V}_{\mathrm{h}(A) \mathrm{e}^{\prime}(2)}\left(r_{B(2)} \mathrm{a}\right)+\mathrm{V}_{\mathrm{e}(1) \mathrm{e}^{\prime}(2)}\left(r_{\mathrm{A}(1)^{\prime}}\right.\right.$, $\left(r_{\mathrm{b}(2)}, \mathrm{a}\right)+\mathrm{V}_{\mathrm{e}(1) h^{\prime}(B)}\left(r_{\mathrm{B}(1)}, \mathrm{a}\right)+\left(\mathrm{V}_{\mathrm{h}(B) \mathrm{h}^{\prime}(A}\right){ }^{(D, a)}+\left(\mathrm{V}_{\mathrm{h}(B) \mathrm{e}^{\prime}(1)}\left(r_{\mathrm{B}(1)}, \mathrm{a}\right)+\mathrm{V}_{\mathrm{e}(2) \mathrm{e}^{\prime}(1)}\left(r_{\mathrm{B}(2)}\right.\right.$, $\left(r_{\mathrm{A}(1)}, \mathrm{a}\right)+\mathrm{V}_{\mathrm{e}(2) h^{\prime}(A)}\left(r_{\mathrm{A}(2),} \mathrm{a}\right)$

$V_{e(1) h(B)}$ is the energy of interaction of the electron $e(1)$ with the hole $h(\mathrm{~B})$; and $V_{e(2) h(A)}$ is the energy of inter- action of the electron $e(2)$ with the hole $h(\mathrm{~A})$. The last- mentioned energies are described by the expressions:

$$
\begin{aligned}
& V_{e(1) h(B)}\left(r_{B(1)}\right)=-\frac{e^{2}}{\varepsilon_{1} r_{B(1)}{ }^{\prime}} \\
& V_{e_{(2)} h_{(A)}}\left(r_{A(2)}\right)=-\frac{e^{2}}{\varepsilon_{1} r_{A(2)}}
\end{aligned}
$$

The energy of Coulomb interaction between the electrons $e(1)$ and $e(2), V_{e(1) e(2)}\left(r_{12}\right)$ is determined by the formula:

$$
V_{e(1) e(2)}\left(r_{12}\right)=-\frac{e^{2}}{\left(\varepsilon_{1} r_{12}\right)}
$$

and the energy of interaction between the holes $h(A)$ and $h(B)$ is described by the expression:

$$
V_{h(A) h(B)}(D, a)=-\frac{e^{2}}{\varepsilon_{1}(D+2 a)}
$$

According to Ref. [2], the major contribution to the energy of the ground state of the exciton (formed by an electron and a hole spatially separated from the electron) is made by the average energy of the Coulomb interaction between the electron and hole $\left\langle R_{0}\left(r_{A(1)}, \tilde{a}\right)\left|V_{e(1) h(A)}\left(r_{A(1)}\right)\right| R_{0}\left(r_{A(1)}, \tilde{a}\right)\right\rangle \quad$ (or) $\left\langle R_{0}\left(r_{B(2)}, \tilde{a}\right)\left|V_{e(2) h(B)}\left(r_{B(2)}\right)\right| R_{0}\left(r_{B(2)}, \tilde{a}\right)\right\rangle$ on the basis of the Coulomb - shaped variational wave functions

$$
\begin{aligned}
& R_{0}\left(r_{\mathrm{A}(1)}, \mathrm{a}\right) \\
& R_{0}\left(r_{A(1)}, \tilde{a}\right)=\tilde{A} \exp \left(-\bar{\mu}(\tilde{a})\left(r_{A(1)} /\left(a_{e x}^{0}\right)\right)\right.
\end{aligned}
$$

Here, $\bar{\mu}(\tilde{a})=\left(\bar{\mu}(\tilde{a}) / m_{0}\right)$ is the variational parameter $\bar{\mu}(a)$ is the reduced exciton effective mass, $m_{0}$ is the electron mass in vacuum) and the normalization constant is

$$
\tilde{A}=\pi^{(-1 / 2)}\left(a_{e x}^{0}\right)^{(-3 / 2)} \bar{\mu}^{(3 / 2)} \exp (\bar{\mu} \tilde{a})\left[2(\bar{\mu} \tilde{a})^{2}+2(\bar{\mu} \tilde{a})+1\right]^{(-1 / 2)}(19)
$$

where $\tilde{a}=\left(a / a_{e x}^{0}\right)$ is the dimensionless QD radius,

$$
a_{e x}^{0}=\frac{2 \varepsilon_{1} \varepsilon_{2}}{\left(\varepsilon_{1}+\varepsilon_{2}\right)} \cdot \frac{\hbar^{2}}{\mu_{o} e^{2}}
$$

is the Bohr radius of a two-dimensional (2D) exciton localized above the planar interface between a semiconductor with the permittivity $\varepsilon_{2}$ and a matrix with the permittivity $\varepsilon_{1}$ (the hole is in motion within the semiconductor, whereas the electron is in the matrix), $e$ is the electron charge $\left.\left.\mu_{0}=m_{\mathrm{e}}{ }^{(1)} m_{h}\right) / m_{\mathrm{e}}{ }^{(1)}+m_{h}\right)$ is the reduced 2D- exciton effective mass.

The above-mentioned feature allows us to retain only the energies of the Coulomb interaction between the electron and hole $\mathrm{V}_{\mathrm{e}(1) h(A)}$ $\left(r_{A(1)},(3)\right.$ and $\mathrm{V}_{\mathrm{e}(2) h(B)}\left(r_{B(2)}\right.$, determined by a formula similar to (3), correspondingly, in the Hamiltonians $\hat{H}_{A(1)}$ (2) and $\hat{H}_{B(2)}[14]$ and to retain only the energy of the interaction between the holes $h(A)$ and $h(\mathrm{~B}) \mathrm{V}_{h(A) h(B)}(D, a)$ [17] in the interaction energy $V_{A B}(D, a)[13]$. At the same time, the energy $V_{A B}(D, a)$ is determined by the formula (17):

$$
V_{A B}(D, a)=V_{h(A) h(B)}(D, a)=-\frac{e^{2}}{\varepsilon_{1}(D+2 a)}
$$

With the above assumptions, the superatom Hamiltonians $\hat{H}_{A(1)}(2)$ and $\hat{H}_{B(2)}(11)$ take the form:

$$
\begin{aligned}
& \hat{H}_{A(1)}=-\frac{\hbar^{2}}{2 \mu} \Delta_{(1)}+V_{e(1) h(A)}\left(r_{A(1)}\right)+E_{g} \\
& \hat{H}_{B(2)}=-\frac{\hbar^{2}}{2 \mu} \Delta_{(2)}+V_{e(2) h(B)}\left(r_{B(2)}\right)+E_{g} .
\end{aligned}
$$

In this case, the quasimolecule Hamiltonian $\hat{\mathrm{H}}_{\mathrm{A}(1)}$ involves the superatom Hamiltonians $\hat{\mathrm{H}}_{\mathrm{A}(1)}(21)$ and $\hat{\mathrm{H}}_{\mathrm{B}(2)}$ (22) as well as the Hamiltonian $\hat{\mathrm{H}}_{\text {int }}$ (15), in which $\hat{\mathrm{H}}_{\mathrm{int}}(12)$, in which the interaction energy $\mathrm{V}_{\mathrm{AB}}(\mathrm{D}, \mathrm{a})$ is determined by formula (20).

On the assumption that the spins of electrons $e$ (1) and $e$ (2) are antiparallel we write the normalized wave function of the singlet ground state of the exciton quasimolecule in the form of a linear combination of wave functions and $\psi_{1}\left(r_{\mathrm{A}(1)}, r_{B(2)}\right)$ and $\psi_{2} r_{A(2),} r_{B(1)},[11-14]$

$\psi_{1}\left(r_{\mathrm{A}(1)}, r_{A(2))}, r_{B(1)}, r_{B(2)}=\left[2\left(1+\mathrm{s}^{2}(\mathrm{D}, \mathrm{a})\right)\right]^{-1 / 2}\left[\psi_{1}\left(r_{\mathrm{A}(1)}, r_{B(2))}+\psi_{2(} r_{A(2),}, r_{B(1)}\right]\right.\right.$ 
Considering that electrons $e(1)$ and $e(2)$ move independently of each other we represent wave functions $\psi_{1}\left(r_{\mathrm{A}(1)}, r_{B(2)}\right)$ and $\psi_{2} r_{A(2),}, r_{B(1)}$ (23) as the product of one-electron variational wave functions $\psi_{\mathrm{A}(1)} r_{A(1)}$ and $\psi_{\mathrm{B}(2)}\left(r_{B(2)}\right)$ and also $\psi_{\mathrm{A}(2)}\left(r_{A(2)}\right)$ and $\psi_{\mathrm{B}(1)}\left(r_{B(1)}\right)$ respectively $[3,11-14]$

$$
\begin{aligned}
& \psi_{1}\left(r_{\mathrm{A}(1)}, r_{\mathrm{B}(2)}\right)=\psi_{\mathrm{A}(1)}\left(r_{\mathrm{A}(1)}\right) \psi_{\mathrm{B}(2)}\left(r_{\mathrm{B}(2)}\right) \\
& \psi_{\mathrm{A}(2)} r_{B(1)=} \psi_{\mathrm{A}(2)}\left(r_{\mathrm{A}(2)}\right) \psi_{\mathrm{B}(1)}\left(r_{B(1)}\right)
\end{aligned}
$$

We represent the one-electron wave functions $\psi_{\mathrm{A}(1)}\left(\mathrm{r}_{\mathrm{A}(1)}\right.$ and $\psi_{\mathrm{B}(2)}$ $\left(r_{B(2)}\right.$ that describe, correspondingly, the electron e(1) localized above the $\mathrm{QD}(\mathrm{A})$ surface and the electron e(2) localized above the $\mathrm{QD}(\mathrm{B})$ surface and the wave functions $\psi_{\mathrm{A}(2)}\left(\mathrm{r}_{\mathrm{A}(2)}\right)$ and $\psi_{\mathrm{B}(1)}\left(\mathrm{r}_{\mathrm{B}(1)}\right)$ that describe, correspondingly, the electron $\mathrm{e}(2)$ localized above the $\mathrm{QD}(\mathrm{A})$ surface and the electron $\mathrm{e}(1)$ localized above the $\mathrm{QD}(\mathrm{B})$ surface as variational Coulomb-shaped wave functions [11]

$$
\begin{aligned}
& \varphi_{A(1)}\left(r_{A(1)}\right)=\tilde{A} \exp \left(-\bar{\mu}(\tilde{a})\left(r_{A(1)} /\left(a_{e x}^{0}\right)\right)\right. \\
& \varphi_{B(2)}\left(r_{B(2)}\right)=\tilde{A} \exp \left(-\bar{\mu}(\tilde{a})\left(r_{B(2)} /\left(a_{e x}^{0}\right)\right)\right. \\
& \varphi_{A(2)}\left(r_{A(2)}\right)=\tilde{A} \exp \left(-\bar{\mu}(\tilde{a})\left(r_{A(2)} /\left(a_{e x}^{0}\right)\right)\right. \\
& \varphi_{B(1)}\left(r_{B(1)}\right)=\tilde{A} \exp \left(-\bar{\mu}(\tilde{a})\left(r_{B(1)} /\left(a_{e x}^{0}\right)\right)\right.
\end{aligned}
$$

Because of the identity of the electrons, the wave function $\psi_{2}\left(r_{\mathrm{A}(2)}\right.$, $r_{\mathrm{B}(1)}(25)$ is equivalent to the wave function $\psi_{1}\left(r_{\mathrm{A}(1)}, r_{\mathrm{B}(2)}(24)\right.$. In (23), the overlapping integral $S(D, a)$ is determined by the formula

$$
S(D, a)=\int d \tau_{1} \psi_{\mathrm{A}(1)}\left(r_{\mathrm{A}(1)} \psi_{\mathrm{B}(1)}\left(r_{\mathrm{B}(1)}\right)\right.
$$

where $d \tau_{1}$ is the volume element of the electron $e(1)$.

In the first approximation, the energy of the exciton quasimolecule singlet ground state is defined by the average value of the Hamiltonian $\hat{\mathrm{H}}(1)$ on the basis of states described by the zero-approximation wave functions $\psi_{s}(23)[11]$

$$
E(D, \bar{\mu}(a, D) a)=\left\langle\psi_{\mathrm{S}}\left(r_{A(1)}, r_{A(2)}, r_{B(1)}, r_{B(2)}\right)|\hat{H}| \psi_{\mathrm{S}}\left(r_{A(1)}, r_{A(2)}, r_{B(1)}, r_{B(2)}\right)\right\rangle
$$

With the explicit form of the wave functions (23) - (29), the energy functional of the exciton quasimolecule singlet ground state takes the form

$$
E_{0}(\tilde{D}, \bar{\mu}(\tilde{a}, \tilde{D}) \tilde{a})=2 E_{e x}(\tilde{a}, \bar{\mu}(\tilde{a}))+\frac{J(\tilde{D}, \bar{\mu}(\tilde{a}, \tilde{D}) \tilde{a})+K(\tilde{D}, \bar{\mu}(\tilde{a}, \tilde{D}) \tilde{a})}{1+S^{2}(\tilde{D}, \bar{\mu}(\tilde{a}, \tilde{D}) \tilde{a})}
$$

Here, $E_{e x}(\tilde{a}, \bar{\mu}(\tilde{a}))$ is the energy functional of the exciton ground state (for the exciton formed from an electron and a hole spatially separated from the electron):

$$
E_{e x}(\tilde{a}, \bar{\mu}(\tilde{a}))=\left\langle\varphi_{A(1)}\left(r_{A(1)}\right)\left|\hat{H}_{A(1)}\right| \varphi_{A(1)}\left(r_{A(1)}\right)\right\rangle
$$

The second term in (32) is a functional $E_{B}(D, a)$ the binding energy of singlet ground state of excitonic quasimolecule. In the functional determined by formula (32), $J(\tilde{D}, \bar{\mu}(\tilde{a}, \tilde{D}) \tilde{a})$ is determined by the expression (here $\tilde{D}=\left(D / a_{e x}^{0}\right)$ )

$$
J(\tilde{D}, \bar{\mu}(\tilde{a}, \tilde{D}) \tilde{a})=\left\langle\varphi_{A(1)}\left(r_{A(1)}\right) \varphi_{B(2)}\left(r_{B(2)}\right)\left|\hat{H}_{i n t}\right| \varphi_{A(1)}\left(r_{A(1)}\right) \varphi_{B(2)}\left(r_{B(2)}\right)\right\rangle
$$

The functional $J(\tilde{D}, \bar{\mu}(\tilde{a}, \tilde{D}) \tilde{a})$ (34) can be represented as the algebraic sum of the functionals of the average energies of Coulomb interaction [11].

In the functional described by $(32), K(\tilde{D}, \bar{\mu}(\tilde{a}, \tilde{D}) \tilde{a})$ is determined by the formula

$$
K(\tilde{D}, \bar{\mu}(\tilde{a}, \tilde{D}) \tilde{a})=\left\langle\varphi_{B(1)}\left(r_{B(1)}\right) \varphi_{A(2)}\left(r_{A(2)}\right)\left|\hat{H}_{i n t}\right| \varphi_{A(1)}\left(r_{A(1)}\right) \varphi_{B(2)}\left(r_{B(2)}\right)\right\rangle
$$

The functional $K(\tilde{D}, \bar{\mu}(\tilde{a}, \tilde{D}) \tilde{a})$ (35) can be represented as the algebraic sum of the functionals of the average energies of the exchange interaction [11].

In the case it spins of $e(1)$ and $e(2)$ electrons are parallel than similarly to the theory of the chemical bond of hydrogen molecule [20] the excitonic quasimolecule, consisting of two QDs is not formed [14]. Therefore, we did not consider this case.

Within the framework of the variational method at a first approximation the total energy of ground singlet state of excitonic quasimolecule is determined by average value of the Hamiltonian $\hat{H}(1)$ for states, which are described by wave functions of the zero approximation $\psi \mathrm{s} r_{\mathrm{A}(1)}, r_{A(2))}, r_{B(1)}, r_{B(2)}(23)[14]$ :

$$
E_{0}(D, a)=2 E_{e x}(a)+E_{B}(D, a)
$$

Where $E_{B}(D, a)$ is the binding energy of singlet ground state of excitonic quasimolecule and the binding energy of $E_{e x}(a)$ of ground state of electron in superatom, found in Ref. [2-9]. The wave function $\psi s \mathrm{r}_{\mathrm{A}(1)}, \mathrm{r}_{\mathrm{A}(2))}, \mathrm{r}_{\mathrm{B}(1)} \mathrm{r}_{\mathrm{B}(2)}$ [21] contains wave functions. The results of a numerical variational calculation of the binding energy $E_{B}(D, a)$ of ground singlet state of excitonic quasimolecule, containing two $\mathrm{CdS}$ QDs with average radii of $\overline{\mathrm{a}}_{1}=4 \mathrm{~nm}$ and $\overline{\mathrm{a}}_{2}=4.4 \mathrm{~nm} \varepsilon_{2}=9.3$, effective hole mass in QD $\left(\mathrm{m}_{\mathrm{h}} / \mathrm{m}_{0}\right.$ is 5)), grown in a matrix of borosilicate glass $\varepsilon_{1}=2$ the electron effective mass in the matrix $\left(\mathrm{m}_{\mathrm{e}}^{(1)} / \mathrm{m}_{0}\right)$ is 0.537$)$ ), which was investigated in experimental work $[5,6]$ are shown in Tables 1 and 2 [14].

In Ref. [5,6], we studied the optical properties of the samples of borosilicate glass, doped with CdS with concentrations from $x \approx 0,003 \%$ to $1 \%$. The average radius $\bar{a}$ of CdS QDs was ranged $\bar{a} \approx 2.0-20 \mathrm{~nm}$.

\begin{tabular}{|c|c|c|c|c|}
\hline $\bar{a}_{1} \mathbf{n m}$ & $E_{e x}\left(\bar{a}_{1}\right) \mathbf{m e V}$ & $\mathbf{D} \mathbf{n m}$ & $E_{B}\left(D, \bar{a}_{1}\right) \mathbf{m e V}$ & $E_{0}\left(D, \bar{a}_{1}\right) \mathbf{m e V}$ \\
\hline 4 & -320 & 1.8 & 0 & -640 \\
\hline 4 & -320 & 2.2 & -27 & -667 \\
\hline 4 & -320 & 2.6 & -28.1 & -668.1 \\
\hline 4 & -320 & 3 & -27.8 & -667.8 \\
\hline 4 & -320 & 4 & -23.2 & -663.2 \\
\hline 4 & -320 & 6 & -9.5 & -649.5 \\
\hline 4 & -320 & 8.4 & 0 & -640 \\
\hline
\end{tabular}

Table 1: Dependence of the binding energy $E_{i}(D, \bar{a})$ and also the total energy $E_{0}\left(D, \bar{a}_{1}\right)$ of the singlet ground state of the excitonic quasimolecule, consisting of two CdS QDs with average radii $\bar{a}_{1}=4 \mathrm{~nm}$ on the distance $D$ between the surfaces of the QD. In this case the binding energies of an electron in a superatoms are $E_{e x}\left(\bar{a}_{1}\right) \cong-320 \mathrm{meV}$.

\begin{tabular}{|c|c|c|c|c|}
\hline $\bar{a}_{2} \mathbf{n m}$ & $E_{e x}\left(\bar{a}_{2}\right) \mathbf{m e v}$ & $\mathbf{D} \mathbf{~ m}$ & $E_{B}\left(D, \bar{a}_{2}\right) \mathbf{m e V}$ & $E_{0}\left(D, \bar{a}_{2}\right) \mathbf{~ m e V}$ \\
\hline 4.4 & -344 & 1.6 & 0 & -688 \\
\hline 4.4 & -344 & 2.2 & -29.4 & -717.4 \\
\hline 4.4 & -344 & 2.48 & -32.8 & -720.8 \\
\hline 4.4 & -344 & 2.6 & -31.9 & -719.9 \\
\hline 4.4 & -344 & 3 & -28.9 & -716.9 \\
\hline 4.4 & -344 & 4 & -24.4 & -712.4 \\
\hline 4.4 & -344 & 6 & -13.2 & -701.2 \\
\hline 4.4 & -344 & 8 & -3.4 & -691.4 \\
\hline 4.4 & -344 & 9.8 & 0 & -688 \\
\hline
\end{tabular}

Table 2: Dependence of the binding energy $E_{0}\left(D, \bar{a}_{2}\right)$ and also the total energy $E_{0}\left(D, \bar{a}_{2}\right)$ of the singlet ground state of the excitonic quasimolecule, consisting of two CdS QDs with average radii $\bar{a}_{2}=4.4 \mathrm{~nm}$ on the distance $D$ between the surfaces of the QD. In this case the binding energies of an electron in a superatoms are $E_{e x}\left(\bar{a}_{2}\right) \cong-344 \mathrm{meV}$. 
At concentrations CdS QD in the order of $x \approx 0,6 \%$ to $\mathrm{x} \approx 1 \%$ the peak, which is $\left(E-E_{g}\right) \approx-712 \mathrm{meV}$ was found in the absorption spectra of the samples at a temperature of $4 \mathrm{~K}$ (where $E_{g}$ is width band gap of CdS QD) [6]. The variational method, used for estimation of the binding energy $E_{B}(\mathrm{D}, \mathrm{a})$ of ground singlet state of excitonic quasimolecule will be valid if the binding energy $E_{B}(\mathrm{D}, \mathrm{a})$ of quasimolecule is small, compared with the binding energy $E_{e x}(a)$ of the ground state of superatom [11-14].

$$
E_{B}(\mathrm{D}, \mathrm{a}) / E_{e x}(a)<<1
$$

Binding energies $E_{B}(\mathrm{D}, \mathrm{a})$ of ground singlet state of excitonic quasimolecule, containing two CdS QDs with average radii of $\bar{a}_{1}=$ $4 \mathrm{~nm}$ and $\bar{a}_{2}=4.4 \mathrm{~nm}$ have a minimum $E_{B}^{(1)}\left(\mathrm{D}_{1}, \bar{a}_{1}\right) \approx \sim-28.1 \mathrm{meV}$ for a distance $D_{1} \cong 2.6 \mathrm{~nm}$ and $E_{B}^{(2)}\left(\mathrm{D}_{2}, \bar{a}_{2}\right) \approx \sim-32.8 \mathrm{meV}$ for a distance $D_{2} \cong 2.48 E_{B}^{(1)}$ and $E_{B}^{(2)}$ correspond to critical temperatures

$\mathrm{Tc}_{(1)} \approx 326 \mathrm{~K}_{\text {and }} \mathrm{Tc}_{(2)} \approx 380 \mathrm{~K}$ ) (Tables 1 and 2 ) [14]. Binding energies of the ground state of electron of superatoms are $E_{e x}\left(\bar{a}_{1}\right) \cong-320 \mathrm{meV}$ and $E_{e x}\left(\bar{a}_{2}\right) \cong-344 \mathrm{meV}$ [9]. In this case energies of singlet ground state (4) of the excitonic quasimolecule are E0 $E_{0}\left(D_{1}, \bar{a}_{1}\right) \cong-668.1 \mathrm{meV}$ $E_{0}\left(D_{2}, \bar{a}_{2}\right) \cong-720.8 \mathrm{meV}$ (Tables 1 and 2). From a comparison of the total energy $\mathrm{E}_{0}\left(\mathrm{D}, \overline{\mathrm{a}}_{2}\right)$ of quasimolecule with a maximum $\left(E-E_{g}\right)$ $\approx-712 \mathrm{meV}$ we get the value of the distance $D_{3} \approx 4.0 \mathrm{~nm}$ between QDs [14]. Criterion [11] of the applicability of used variational estimation of the binding energy $E_{B}(\mathrm{D}, \mathrm{a})$ of the quasi-molecule is implemented $E_{B}^{(1)}\left(D_{1}, \bar{a}_{1}\right) / E_{e x}\left(\bar{a}_{1}\right), E_{B}^{(2)}\left(D_{2}, \bar{a}_{2}\right) / E_{e x}\left(\bar{a}_{2}\right) \cong 0.09$.

With increasing of QDs radius $a$ the binding energy $E_{e x}(a)$ of the ground state of electron in a superatom increases [2-9]. The average size of the state of electron in the superatom decreases. Therefore the distance, for which the square of the overlapping integral $S(D, a)$ of the one-electron wave functions takes on a maximum value. Also it decreases with increasing of QD radius $a$, and so the distance between surfaces of QD $D_{2}$ is less than $D_{1}$. As a result, with increasing of QD radius $a$ also the maximum value of the binding energy of singlet ground state of excitonic quasimolecule $E_{B}(D, a)$ increases (i.e $E_{\mathrm{B}}{ }^{(2)}$ more $E_{\mathrm{B}}{ }^{(1)}$. In this case, criterion [11] of the applicability of used variational estimation of the binding energy $E_{B}(D, a)$ of the quasimolecule is implemented [14].

Thus, proposed model of excitonic quasimolecule [14] let us to explain the optical properties of nanosystems, consisting of CdS QDs, grown in a borosilicate glass matrix $[5,6]$ in particular, the appearance of a peak $\left(E-E_{g}\right) \approx-712 \mathrm{meV}$ in the absorption spectra of the samples at 4 $K$. It should be noted that the binding energy $E_{e x}(a)$ of the ground state of an electron in superatom [2,9] can be applicable only for values of binding energy $E_{e x}(a)$ for which the inequality

$$
\left(\left(E_{e x}(a)-E_{g}\right)<<\mathrm{V}\right.
$$

is correctly, where $\nabla V$ is the depth of the potential well for electron and hole in a QD. In the experimental work [5] it found that for CdS QDs with radii of $a \leq 20 \mathrm{~nm} \nabla V=2.5 \mathrm{eV}$. This condition (38) makes it possible to consider the motion of electron and hole in the superatom, using QD model as an infinitely deep potential well [2-9] (Figure 1). In the Hamiltonian (1) of quasimolecule members, causing fluctuations of QD in quasimolecule were not considered. The obtained quasimolecule binding energies (Tables 1 and 2) are significantly higher than typical energy of QD fluctuations. Therefore, it is not adjusting for QD in this quasimolecule model is warranted.

From Tables 1 and 2, it is following that the excitonic quasimolecule, consisting of two QD occurs, starting from the distance between surfaces of QDs $D \geq D_{C}^{(1)} \cong \sim 1.8$ for QD with a radius of $\bar{a}_{1}=4 \mathrm{~nm}$ and $D \geq D_{C}^{(1)} \cong 1.6 \mathrm{~nm}$ for QD with a radius of $\bar{a}_{2}=4.4 \mathrm{~nm}$ [14]. The formation of such quasimolecule is threshold character. It is possible only for nanosystems, containing QD with average radii of $\bar{a}_{1}$ and $\bar{a}_{2}$ in which the distance $D$ between surfaces of QDs exceeds some critical distance $\mathrm{D}_{c}{ }^{(1)}$. The existence of such critical distance $\mathrm{D}_{c}{ }^{(1)}$. is associated with dimensional quantum effect, for which a decreasing of distance $D$ between surfaces of QDs led to decrease in the interaction energies of electrons and holes in the Hamiltonian (1) of quasimolecule can not compensate the increase in the kinetic energy of electrons. With the increase in the distance $D$ between surfaces of QDs, starting from values $D \geq D_{C}^{(1)} \cong 8.4 \mathrm{~nm}$ for QD with a radius of $\bar{a}_{2}=4.4 \mathrm{~nm}$ and $D_{C}^{(2)} \cong 9.8 \mathrm{~nm}$ for QD with a radius of $\bar{a}_{2}=4.4 \mathrm{~nm}$ the excitonic quasimolecule splits into two superatoms.

Thus, the excitonic quasimolecule of nanosystem may occur when the condition $\mathrm{D}_{c}^{(1)} \leq \mathrm{D} \leq \mathrm{D}_{c}^{(2)}$ is realized (Tables 1 and 2) [14]. In addition, quasimolecule can exist only at temperatures below a certain critical temperature $\mathrm{Tc}_{(1)} \approx 326 \mathrm{~K}$ and $\mathrm{Tc}_{(2)} \approx 380 \mathrm{~K}$ ) Biexcitons arose in CdS single-crystal with a binding energy $E_{b} \approx 0.59 \mathrm{mev}$ [11].

The binding energies $E_{\mathrm{B}}{ }^{(1)}$ and $E_{\mathrm{B}}{ }^{(2)}$ of excitonic quasimolecule are significant exceeding $E_{\mathrm{B}}$ almost two orders of magnitude. Apparently the latter fact opens the possibility of observing such excitonic quasimolecules at room temperature. The energy of the exchange interaction between electrons and holes main contributes to the binding energy of excitonic quasi-molecule, which is significantly greater than that for energy of the Coulomb interaction between electrons and holes (i.e., their ratio $\leq 0,11)$. The estimations of the binding energy $E_{B}(D, a)$ of the singlet ground state of quasi-molecule are variational and may give low values of the binding energy $E_{B}(\mathrm{D}, \mathrm{a})$ and $E_{\mathrm{B}}{ }^{(1)}$.

\section{Conclusion}

The binding energies of the exciton quasimolecule consisting of two CdS QDs acquires an anomalously high value that exceeds the bond energy $E_{B}$ of the biexciton in CdS by almost two orders of magnitude. Such an effect opens up the possibility of using the exciton quasimolecules as active medium in nanolasers emitting in the infrared region and operating on exciton transitions at room temperatures in the elementary base of quantum nanocomputers $[17,18,21]$. The presented results demonstrate the fundamental possibility of creating novel quasi atomic nanosystems in the form of exciton quasimolecules, including natural systems with new physical characteristics $[3,11$ 14]. On their basis it is possible to construct new nanosystems or quasicrystals in which control of the symmetry and lattice constant will make it possible to realize unique physical effects and phenomena and to create new principles in materials behavior.

\section{References}

1. Ashoori RC (1996) Electrons in artificial atoms. Nature 379: 413-415.

2. Pokutnyi SI (2013) On an exciton with a spatially separated electron and hole in quasi-zero- dimensional semiconductor nanosystems. Semi conductors 47 : 791-798.

3. Pokutnyi SI (2014) Theory of excitons and excitonic quasimolecules formed from spatially separated electrons and holes in quasi-zero-dimensional nanosytems. Optics 1: 10-21.

4. Yakimov Al, Dvurechensky AV (2001) Effects of electron-electron interaction in the optical properties of dense arrays of quantum dots Ge/Si. JETP 119: 574-589.

5. Grabovskis V, Dzenis Y, Ekimov A (1989) Photo ionization of semiconductor microcrystals in glass. Sov Phys Solid State. 31: 272-275.

6. Bondar N (2010) Photoluminescence quantum and surface states of excitons in ZnSe and CdS nano clusters. Journal of Luminescence 130: 1-7.

7. Ovchinnikov OV, Smirnov MS, Shatskikh TS (2014) Spectroscopic investigation of colloidal CdS quantum dots methylene blue hybrid associates. J Nanopart Res 16: 2286-2292. 
Citation: Pokutnyi SI (2016) Excitonic Quasimolecules in Nano Systems of Semiconductor and Dielectric Quantum Dots. Mod Chem appl 4: 188. doi: 10.4172/2329-6798.1000188

Page 6 of 6

8. Dzyuba VP, Kulchin YN, Milichko VA (2013) Quantum size states of a particle inside the nanospheres Advanced Material Research A 677: 42-48.

9. Pokutnyi SI (2013) Binding energy of the exciton with a spatially separated electron and hole in quasi-zero-dimensional semiconductor nanosystems. Technical Physics Letters 39: 233-235.

10. Pokutnyi SI, Kulchin YN, Dzyuba VP (2015) Binding energy of excitons formed from spatially separated electrons and holes in insulating quantum dots. Semiconductors 49: 1311-1315.

11. Pokutnyi SI (2013) Biexcitons formed from spatially separated electrons and holes in quasizero dimensional semiconductor nanosystems Semiconductors 47: $1626-1635$

12. Pokutnyi SI, Salejda W (2015) Theory of excitons and excitonic quasimolecules formed from spatially separated electrons and holes in quasi-zero-dimensional nanostructures. pp: 10-21.

13. Pokutnyi SI, Kulchin YN, Dzyuba VP (2016) Excitonic quasimolecules in quasizero-dimensional nanogeterostructures. Theory Pacific Science Review A 17: $11-13$
14. Pokutnyi SI (2016) Quantum chemical analysis of system consisting of two CdS quantum dots. Theoretical and Experimental Chemistry 52: 27-32.

15. Lalumiure K, Sanders B, VanLoo F (2013) Input - output theory for waveguide QED with an ensemble of in homogeneous atoms. Phys Rev A 88: 43806-43811.

16. VanLoo F, Fedorov A, Lalumiure K (2013) Photon-mediated interactions between distant artificial atoms. Science 342: 1494-1496.

17. Lozovik YE (2014) Electronic and collective properties of topological insulators Advanc Phys Scienc 57: 653-658.

18. Valiev K (2005) Quantum computers and quantum computing. Advanc Phys Scienc 48: 1-22.

19. Pokutnyi SI (2007) Exciton states in semiconductor quantum dots in the framework of the modified effective mass method. Semiconductors 41: 1323-1328.

20. Schiff L (1955) Quantum Mechanics. McGraw-Hill Book Company Inc., New York, Toronto, London.

21. Pokutnyi SI, Ovchinnikov OV, Kondratenko TS (2016) Absorption of light by colloidal semiconductor quantum dots. J Nanophotonics 10: 033506-1-033506-9. 